\title{
LETTER
}

doi:10.1017/\$1041610216000119

\section{Alfentanil anesthetic augmentation lengthens seizure duration in electroconvulsive therapy with older people}

Electroconvulsive therapy (ECT) prescription rates rise with age, making it important that treatments be made as effective and safe as possible (Plakiotis et al., 2012). Older people are vulnerable to posttreatment confusion and to subsequent deficits in attention, new learning, and autobiographical memory (Gardner and O'Connor, 2008). Strategies to minimize cognitive side-effects include unilateral electrode placement and stimulus dose titration whereby electrical charge is individually calibrated to seizure threshold (Sackeim et al., 2000). It remains the case, however, that threshold levels typically rise over the treatment course, leading to an increase both in delivered charge and the risk of adverse sequelae.

Anesthetic agents impact on ECT delivery and warrant consideration as tools to minimize risk. Propofol is used widely for ECT and other brief procedures because of its rapid onset of action, stable cardiovascular profile, and speedy recovery. Against this, its anticonvulsant properties make it more difficult to induce a therapeutic, supra-threshold seizure. When this happens, the electrical charge must be increased, with predictable consequences (Loo et al., 2010).

The potent, rapid acting opioids, remifentanil, and alfentanil, look promising as adjuvant anesthetic induction agents given their lack of anticonvulsant action and their attenuation of the sympathetically induced tachycardia and hypertension that accompany ECT. In a systematic review, remifentanil was associated in most studies with longer seizures, a higher postictal suppression index and lower pulse rate and blood pressure than propofol, methohexital, or thiopentone (Chen, 2011). Findings with respect to seizure threshold were mixed. Seizures tended to be longer when remifentanil was used alone, or with lower doses of the primary anaesthetic agent, but not when the dose of the primary anesthetic was maintained. It seems likely, therefore, that the longer seizures resulted mostly from a reduction in the dose of other agents. There is a trend now to combine an opioid medication with a lower than usual dose of propofol with the goal of minimizing electrical charge, cardiovascular stress, and cognitive side effects. These advantages must be weighed against a slower recovery time (Loo et al., 2010).

Most published reports have focused on remifentanil's effectiveness in younger adults. It cannot be assumed that findings apply equally to alfentanil or to treatment of older people who tend to have higher seizure thresholds and lower amplitude, more disorganized seizures (Plakiotis and O'Connor, 2011). We therefore report here on the use of alfentanil in the first 14 patients of an aged mental health service to be switched at their consultant's request from propofol alone to a mixture of propofol and alfentanil with a view to reducing electrical charge because of concerns about escalating charge and actual or likely cognitive side effects. Consent to publish de-identified clinical data was obtained from the relevant health ethics committee.

Twelve of the 14 patients were female. Their ages ranged from 65 to 84 years (mean 75.4 years) and all were depressed. The first mixed agent treatment session described here ranged from the 2 nd to 13 th in a course (mean 8.4). There were no simultaneous changes in psychotropic medications, electrode placement (eight right unilateral, six bitemporal), or doses of suxamethonium. Mean doses of propofol were deliberately reduced from $70.4 \mathrm{mg}$ in the previous session to $44.6 \mathrm{mg}$. Alfentanil doses ranged from 250 to $1000 \mathrm{mcg}$, mean $594 \mathrm{mcg}$ (4.1 to $14.1 \mathrm{mcg} / \mathrm{kg}$, mean $8.9 \mathrm{mcg} / \mathrm{kg}$ ). Treatments were delivered by Thymatron System IV machines.

Visually gauged seizures lengthened in duration with alfentanil from a mean of 34.8 secs in the previous session to $49.8 \operatorname{secs}(t=-2.45$, df $11, p=$ $0.03)$. Data were missing in two cases. No significant differences were identified in machine generated parameters including seizure amplitude, coherence, or suppression. There were no adverse sequelae.

These findings are in line with previous studies of alfentanil (Akcaboy et al., 2005; Dinwiddie and Isenberg, 1995; Nguyen et al., 1997), suggesting that older people might benefit as much from opioid supplementation as younger ones. The lack of effect on other seizure characteristics most probably reflects a lack of statistical power but, in any event, the link between seizure characteristics and therapeutic benefit is unclear (Plakiotis and O'Connor, 2011). It still needs to be demonstrated though that longer seizures reflect a lower convulsive threshold meaning that electrical charge can be reduced consistently, granting a more favorable cognitive outcome. Future studies should track electrical, affective, and cognitive measures 
over the whole of a treatment course to confirm that our pilot findings translate to real benefits for older patients.

\section{Conflict of interest}

None.

\section{References}

Akcaboy, Z. N. et al. (2005). Effects of remifentanil and alfentanil on seizure duration, stimulus amplitudes and recovery parameters during ECT. Acta Anaesthesiologica Scandinavica, 49, 1068-1071.

Chen, S. T. (2011). Remifentanil: a review of its use in electroconvulsive therapy. Fournal of ECT, 27, 323-327.

Dinwiddie, S. H. and Isenberg, K. E. (1995). Combined alfentanil-methohexital anesthesia in electroconvulsive therapy. Convulsive Therapy, 11, 170-176.

Gardner, B. K. and O'Connor, D. W. (2008). A review of the cognitive effects of electroconvulsive therapy in older adults. Fournal of ECT, 24, 68-80.

Loo, C. K., Kaill, A., Paton, P. and Simpson, B. (2010). The difficult-to-treat electroconvulsive therapy patient: strategies for augmenting outcomes. Fournal of Affective Disorders, 124, 219-227.

Nguyen, T. T., Chhibber, A. K., Lustik, S. J., Kolano, J. W., Dillon, P. J. and Guttmacher, L. B. (1997). Effect of methohexitone and propofol with or without alfentanil on seizure duration and recovery in electroconvulsive therapy. British Fournal of Anaesthetics, 79, 801-803.

Plakiotis, C. and O'Connor, D. W. (2011). Ictal electroencephalographic measures in electroconvulsive therapy. Current Psychiatric Reviews, 7, 256-280.

Plakiotis, C., George, K. and O'Connor, D. W. (2012). Has ECT utilisation remained stable over time? A decade of ECT service provision in Victoria, Australia. Australian and New Zealand Fournal of Psychiatry, 46, 522-531.

Sackeim, H. A. et al. (2000). A prospective, randomized, double-blind comparison of bilateral and right unilateral electroconvulsive therapy at different stimulus intensities. Archives of General Psychiatry, 57, 425-434.

\section{Craig D'Cunha, ${ }^{1}$ Christos Plakiotis ${ }^{1}$} AND DANIEL W. O'CONNOR ${ }^{1,2}$

${ }^{1}$ Department of Psychiatry, Monash University, Melbourne, Victoria, Australia

${ }^{2}$ Institute for Health and Ageing, Australian Catholic University, Melbourne, Victoria, Australia 